\title{
Acute inflammatory demyelinating polyradiculoneuropathy following varicella
}

\author{
J.M.K. Murthy
}

Department of Neurology, The Nizam's Institute of Medical Sciences, Panjagutta, Hyderabad 500482 , India

\begin{abstract}
Summary: Four cases of acute inflammatory demyelinating polyradiculoneuropathy following varicella are described. The role of immunosuppression as a contributing factor in triggering an autoimmune disease of the peripheral nervous system following viral infection is discussed.
\end{abstract}

\section{Introduction}

Acute inflammatory demyelinating polyradiculoneuropathy (AIDP) or Guillain-Barré syndrome (GBS) is a rare complication of varicella infection, the calculated rate being one case per 15,000 cases of varicella. ${ }^{1}$ AIDP constituted $7 \%$ of neurological complications of varicella studied by Miller et al. ${ }^{2}$ Twenty six cases of AIDP in association with varicella have been reported up to 1984.' Four cases of AIDP following varicella were seen over a period of 8 years, during which period a total of 62 cases of AIDP were seen.

\section{Case reports}

Case 1

A boy aged 10 years was admitted for weakness of all four limbs of 4 days duration. Eight days before the onset of these symptoms he had fever associated with rash characteristic of chicken pox. On examination he had still crusted lesions of chicken pox. Muscle tone was reduced in all the four limbs and the power was graded at $2-3 / 5$ in the limbs. Tendon reflexes were not elicitable. Perception for all modalities of sensation was preserved. Cerebrospinal fluid (CSF) analysis showed elevated proteins, $68 \mathrm{mg} / \mathrm{dl}$. Electrophysiological studies showed abnormalities. The distal latencies were 15.1 millisec (normal $<3.5$ ) and 16.1 millisec (normal <4.4) in the median and common peroneal nerves respectively. The compound muscle action potential (CMAP) was polyphasic and dispersed, the amplitudes were much reduced $1.8 \mathrm{mV}$ and $0.8 \mathrm{mV}$ from abductor pollicis brevis and extensor

Correspondence: J.M.K. Murthy M.B.B.S., M.D. 10-5-3/2/7 Masab Tank Hyderabad 500 028. India.

Accepted: 23 April 1987 digitorum brevis (normal $>6.2$ and 3.3). The motor conduction velocities (MCV) $15.3 \mathrm{~m} / \mathrm{sec}$ (normal $>43.2$ ) in the median nerve and $14.2 \mathrm{~m} / \mathrm{sec}$ (normal $>40.3$ ) in the common peroneal nerve. The sensory action potential of the median nerve measured $15 \mu \mathrm{v}$ (normal $>10.2$ ) in amplitude and the peak latency was 3.8 millisec (normal <3.4). No proper sensory action potential could be recorded from the sural nerve (technical). He made a good recovery.

\section{Case 2}

A 62 year old man was admitted with weakness, numbness and paraesthesiae of all the four limbs. He developed these symptoms 8 days after chicken pox. He was normotensive and skin showed crusted lesions of chicken pox. Muscle tone was reduced in all the four limbs, power grading showed grade $2 / 5$ power in all the group of muscles in the lower limbs and $3 / 5$ in upper limb muscles. Tendon reflexes were not elicitable and plantars were down going. Perception for all sensory modalities was diminished below the elbow in upper limbs and below the knee in lower limbs, more so for position and vibration sensations. CSF protein was $60 \mathrm{mg} / \mathrm{dl}$. During his hospital stay he had progression of the disease resulting in bulbar and respiratory muscle paralysis. He was put on respiratory assistance. $\mathrm{He}$ had wide fluctuations in blood pressure due to associated autonomic neuropathy and died.

\section{Case 3}

An 8 year old boy was admitted for weakness of all the four limbs of one month duration. The weakness appeared acutely, 10 days after chicken pox. He had bilateral facial paralysis. Muscle power grading showed grade $0 / 5$ power in proximal muscles and grade $2 / 5$ power in the distal group of muscles. The 
deep reflexes were absent. No sensory deficit could be detected. CSF analysis showed $120 \mathrm{mg} / \mathrm{dl}$ of protein. Electromyography of distal muscles showed occasional fibrillation potentials and positive sharp waves. Motor conduction velocities in the median and common peroneal nerves were $39.8 \mathrm{~m} / \mathrm{sec}$ and $32 \mathrm{~m} /$ $\mathrm{sec}$ respectively. Distal latencies were 4.5 millisec and 6.2 millisec in the median and common peroneal nerves respectively. The amplitudes of CMAPs from abductor pollicis brevis and extensor digitorum brevis were $3.5 \mathrm{mV}$ and $2 \mathrm{mV}$. Sensory action potential from median nerve measured $15 \mu \mathrm{v}$ and the peak latency was 2.1 millisec. Sensory action potential of sural nerve measured $10 \mu \mathrm{V}$ and the peak latency was 3.6 millisec. He showed gradual improvement and at one year he was able to walk independently.

\section{Case 4}

A 52 year old man developed weakness of all the four limbs and inability to close his eyes 8 days following chicken pox. He had bilateral facial paralysis, more on the right side. The power in the lower limbs was graded $3 / 5$ and $4 / 5$ in the upper limbs. Deep reflexes were absent. Perception for vibration and sense of position was decreased below the knees. Motor conduction studies showed $44.4 \mathrm{~m} / \mathrm{s}$ in the median nerile and 23.4 millisec in the common peroneal nerve. The CMAPs from abductor pollicis brevis and extensor digitorum brevis were of $4 \mathrm{mV}$ and $2 \mathrm{mV}$ amplitude respectively. Sensory action potentials of median and

\section{References}

1. Arnson, B.G.W. Acute inflammatory demyelinating polyradiculoneuropathy. In: Dyck, P.J., Thomas, P.K., Lambert, E.H. \& Bunge, R. (eds) Peripheral Neuropathy, Vol 2, Second edition. W.B.Saunders, Philadelphia, 1984, pp 2050-2100.

2. Miller, J.D., Stanton, J.B. \& Gibbons, J.L. Para infectious encephalomyelitis and related syndromes. A critical review of the neurological complications of certain specific fevers. $Q J$ Med 1956, 25: 427-505.

3. McKendell, R.R. \& Klawans, W.L. Nervous system complications of varicella-zoster virus. In: Vinken, P.J. \& Bruyn, G.W. (eds) Handbook of Clinical Neurology, Vol 34. North-Holland Publishing Co., Amsterdam, 1978, pp 161-383

4. Raman, P.T. \& Taori, G.M. Prognostic significance of electrodiagnostic studies in the Guillain-Barré syndrome. J Neurol Neurosurg Psychiatry 1976, 39: 163170.

5. Wybran, J. \& Fudenberg, H.H. Thymus derived rosette- sural nerves measured $14.5 \mu \mathrm{V}$ and $8 \mu \mathrm{V}$ respectively, the corresponding peak latencies were 2.7 millisec and 3.8 millisec. He made a good recovery.

\section{Discussion}

Symptoms of involvement of the nervous system following varicella develop between the fifth and twentieth day after the appearance of the rash, usually in the first half of the second week. ${ }^{2,3}$ There are no neurological features which serve to distinguish the syndrome of AIDP seen in association with varicella from that seen in other clinical settings. The prognosis is good. ${ }^{3}$ The electrophysiological findings were suggestive of a demyelinating type of neuropathy such as AIDP. ${ }^{4}$

Variable degrees of immunosuppression, particularly cell-mediated immunity are known to follow viral infections, ${ }^{5}$ Depression of tuberculin reactivity occurs in chicken pox. ${ }^{6}$ Lisak et al. ${ }^{7}$ suggested the role of immunosuppression as a contributing factor in triggering an autoimmune disease of the peripheral nervous system. It is possible that viral infections may reduce suppressor $\mathrm{T}$ cell activity thereby leading to proliferation of those few $T$ and $B$ lymphocytes whic $B$ are normally present in humans and recognise nervous system antigen. ${ }^{8}$ Reduction of the suppressor $\mathrm{T}$ cee subpopulation in patients with AIDP $^{9}$ is consisten with this hypothesis. forming cells in various human disease states. Cancer, lymphoma, bacterial and viral infections and other diseases. J Clin Invest 1973, 52: 1026-1032.

6. Starr, S. \& Berkovich, S. The depression of tuberculin reactivity during chicken pox. Pediatrics 1964, 33: 769 772.

7. Lisak, R.P., Mitchell, M., Zweiman, B., Orrechio, E. \& Asbury, A.K. Guillain-Barré syndrome and Hodgkin's disease: Three cases with immunological studies. Ann 음 Neurol 1977, 1: 72-78.

8. Hughes, R.A.C. \& Winer, J.B. Guillain-Barré syndrome. In Matthews, W.B. \& Glaser, G.H. (eds) Recent Advances in Clinical Neurology. Churchill Livingstone, Edin- $\sigma$ burgh, 1984, pp 19-49.

9. Hughes, R.A.C., Aslan, S. \& Gray, I.A. Lymphocyte subpopulation and suppressor cell activity in acute $\omega$ polyradiculoneuritis (Guillain-Barré syndrome). Clin Exp Immunol 1983, 51: 448-454. 